\title{
VISUAL ACUITY ASSESSMENT
}

\author{
J. R. WEATHERILL \\ Bradford
}

Tests of visual function in cataract patients are performed in order to answer two questions: (1) To what extent is the cataract responsible for the patient's symptoms? (2) Will removal of the cataract improve the patient's sight? To answer the first question the optical qualities of the eye are assessed, and to answer the second tests are employed which assess retinal and neural function and which are relatively unaffected by the optical degradation caused by opacities in the media. At Bradford we employ the tests listed in Table I.

\section{Snellen Acuity}

Although the Snellen acuity test measures the function of only the central $1-2^{\circ}$ in monochrome at $100 \%$ contrast, it is nonetheless the 'gold standard' by which ophthalmologists judge and are judged. It is universally recognised and enjoys a legal status. Despite its limitations, in the vast majority of patients the pattern and degree of cataract are consistent with the Snellen acuity which experience has taught us to expect, and the decision as to whether to operate or not is straightforward. In a minority of patients the Snellen actuity does not correspond with the observed degree of cataract. If the acuity is worse than expected, retinal function tests are employed; if the Snellen acuity is much better than the patient's symptoms would suggest, tests of the optical system may demonstrate the cause of the difficulties.

A more accurate form of the Snellen chart is the FerrisBailey Logmar ${ }^{1}$ chart, which we have used for monitoring the progress of cataract. Its features are: (1) there are an equal number of letters on each line, (2) the letters have been chosen for equal legibility, (3) the increase in size of the letters is on a logarithmic scale, and (4) the working distance is $4 \mathrm{~m}$. This chart is widely used in vision research and is no more difficult for the patient than the Snellen chart. In the interests of reproducibility and accuracy the Logmar chart should replace the Snellen chart as the standard acuity test.

\section{Reading Vision}

There is a need for a rigorous test of near vision. It is well

Correspondence to: J. R. Weatherill, Department of Ophthalmology, Bradford Royal Infirmary, Bradford, West Yorkshire BD9 6RJ, UK. recognised that patients with macular dysfunction have difficulty in reading out of proportion to their Snellen acuity. This difficulty is probably due to parafoveal scotomas which prevent the whole word being seen at the same time. If a patient reports that the main problem is with reading, it is helpful to observe whether the patient holds the page steadily or keeps moving it to avoid scotomas. With current reading tests patients with poor macular function can read small print for brief periods, particularly if they are used to reading and recognise the overall shape of the words. A Logmar equivalent for near vision comprising random words of equal length and composed of letters without either ascenders or descenders, read under controlled conditions of illumination and at a standard distance, would provide a more accurate measure of macular function. It would possibly also be helpful in the management of conditions such as diabetic maculopathy in which patients often report improvement in near vision after treatment although the Snellen acuity is little altered.

\section{Contrast Sensitivity}

For many years contrast sensitivity has been a research tool and each department has devised its own techniques, but for clinical purposes the Pelli-Robson chart, in which letters of uniform size are displayed in decreasing contrast, gives reproducible results. High-frequency closely spaced lines can only be seen at high contrast and this is equivalent to the Snellen acuity. Low-frequency lines can be seen with less contrast. A high degree of contrast sensitivity from low to high frequency is essential for normal vision. One of the most important functions of sight is to recognise faces. One of the commonest complaints of cat-

Table I. Tests of visual function used at Bradford

\begin{tabular}{lcc}
\hline & Optical test & Retinal test \\
\hline Snellen acuity & + & + \\
Reading vision & + & ++ \\
Contrast sensitivity (CS) & ++ & + \\
CS + Glare & +++ & + \\
CS + 'Noise' & + & ++ \\
Interferometry & + & ++ \\
Threshold displacement & 0 & ++ \\
\hline
\end{tabular}

The symbols indicate the sensitivity of each test to optical degradation and retinal function. 


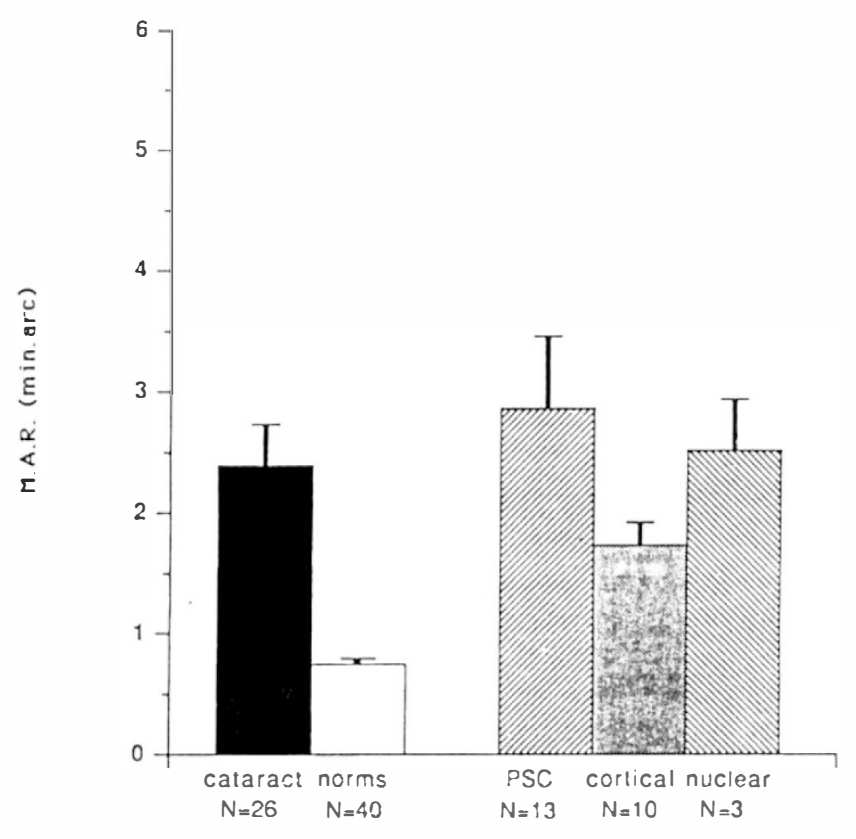

Fig. 1. Minimum angle of resolution (M.A.R.) for both the cataract and the normative group. The cataract group is further subdivided into posterior subcapsular (PSC), cortical and nuclear types. Standard error bars are shown.

aract patients is difficulty in recognising faces. In order to do this low-frequency contrast sensitivity is needed to reveal the patterns of light and shade which correspond to the facial features; patients who have lost low-frequency contrast sensitivity but retain high-frequency contrast sensitivity perceive only the sharp boundaries at the edges of major features.

Using the Bristol questionnaire devised by Lowe, Elliott ${ }^{2}$ has demonstrated a much closer correlation between a patient's perceived disability and loss of contrast sensitivity than between perceived disability and Logmar acuity. The questionnaire comprises 20 questions relating to hobbies, mobility, reading, television watching and recognition of faces. The contrast sensitivity was measured on the Pelli-Robson chart. The patients were tested monocularly and binocularly and a glare test was included. A multivariate analysis showed that:

1. Snellen visual acuity did not correlate well with outdoor tasks but did correlate with reading difficulty. The visual acuity of the worse eye gave the closer correlation. This is an example of binocular inhibition.

2. Poor binocular contrast sensitivity correlated well with disability outdoors as well as with difficulty with near vision tasks.

Contrast sensitivity measurements are therefore a more reliable guide to the likely benefits of cataract surgery than are Snellen acuity measurements. Even more important is the finding that the blurred image from the worse eye inhibits contrast sensitivity in the better eye. ${ }^{3}$ It follows that during normal binocular viewing patients who have uniocular cataract are suffering loss of vision in their normal eye and should therefore be offered cataract surgery. The results of contrast sensitivity testing clearly demonstrate visual handicap which is not necessarily apparent from the Snellen acuity.

\section{GLARE AND CONTRAST SENSITIVITY}

If the patient is asked to view a contrast sensitivity or Snellen chart against a bright background the acuity drops further in the presence of lens opacities. This accords with clinical experience that glare can be a major problem even when the Snellen acuity is good. The converse of glare testing is the pinhole test in which a fine beam of light is guided through a clear part of the lens: because of the narrowness of the beam, refractive blur circles are at a minimum. Under these conditions some patients with dense opacities can see well, thus demonstrating the integrity of the macula.

\section{'NOISE' CHARTS}

It is well known that contrast sensitivity is reduced in retinal and neural disorders as well as by cataract, and in order to improve the specificity of contrast sensitivity tests a chart has been devised to differentiate between loss of contrast sensitivity from lens opacities and loss of contrast sensitivity from retinal and neural disorders. The auditory system can recognise a tune or conversation against a background of random noise. Similarly, the visual system can identify meaningful signals against a random background. The hypothesis is that if a patient is tested on a clean Pelli-Robson chart and on one splattered with blotches ('background noise'), then the results should be similar if the loss of contrast sensitivity is due to cataract but will show a greater loss on the 'noisy' chart if the retinal and neural systems are impaired. This test is at an early stage of development and reproducible results are not yet available.

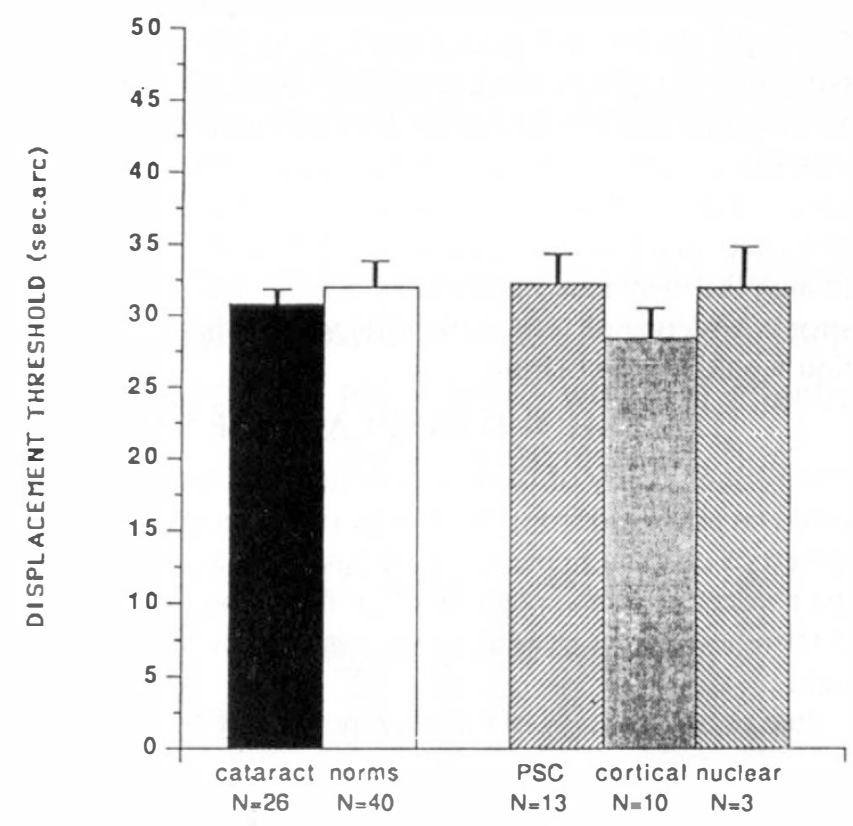

Fig. 2. Displacement threshold in seconds of arc for both the cataract and the normative group. The cataract group is further subdivided into posterior subcapsular (PSC), cortical and nuclear types. Standard error bars are shown. 


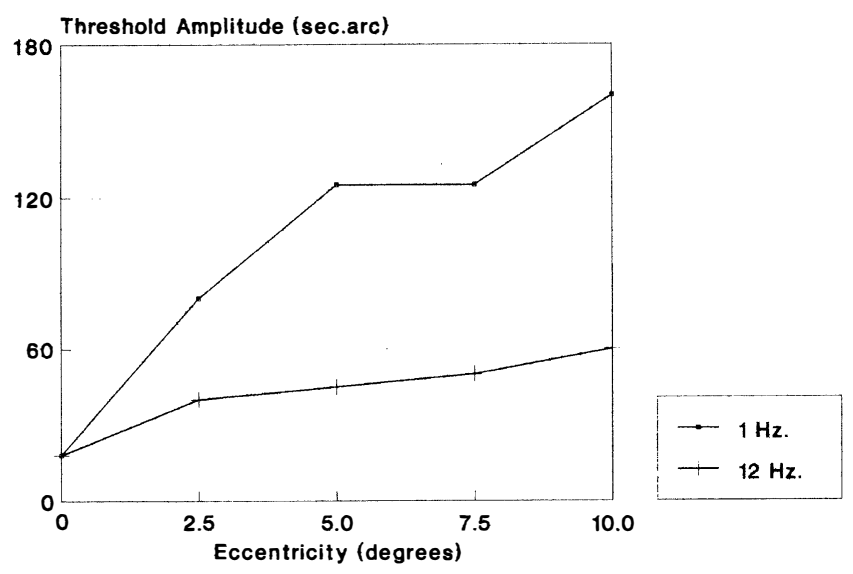

Fig. 3. The minimum displacement or threshold displacement of a target which can be detected at increasing degrees of eccentricity from fixation. The upper line plots the response of a target oscillating at $1 \mathrm{~Hz}$ and the lower line plots the response to the same target oscillating at $12 \mathrm{~Hz}$.

\section{LASER INTERFEROMETRY}

The principle of laser interferometry ${ }^{4}$ is to project two beams of coherent light on to the retina to generate an interference pattern which is perceived as alternating red and black stripes the orientation of which can be varied. The coherent beams are said to be relatively resistant to degradation by opacities and in many cases there is a good correlation between predicted and actual post-operative acuity. Not surprisingly the more dense the cataract the less accurate the prediction. In practice we found that it was necessary to find a clear gap in the cataract in order to achieve satisfactory interference fringes. A more serious problem with this technique is that false positives, that is over-optimistic predictions, occur if pathology is confined to the foveal and parafoveal regions. The reasons for these false positives are: (1) the size of the stimulus, which is $5.5^{\circ}$ in the Rodenstock instrument, and (2) the striped pattern used, which is a more primitive visual stimulus than an optotype and can be detected by arrays of distorted or damaged receptors which would not detect the more complex image. In practice the test is most useful in the negative sense. If a patient produces a very poor response even though the media are not densely opaque then this is very strong evidence of retinal pathology and cataract extraction would not be helpful.

\section{THRESHOLD DISPLACEMENT}

The vernier acuity of the visual system is far higher than could be predicted from the density of the receptors and is therefore called a hyperacuity. It has been suggested that the perception of the position of an image on the retina is determined by the 'centre of gravity' or centroid of its light distribution.

Just as tiny degrees of misalignment can be detected so similarly very small movements can be perceived. A displacement threshold is the determination of the smallest change of position of an object which gives rise to the sensation of movement. Low-frequency oscillation of $40^{\prime \prime}$ can be detected in an isolated luminous target, but if stationary reference lines are added to the field of view then displacements of $10^{\prime \prime}$ can be detected. The oscillation is seen as a displacement from the reference bars and not as an inherent movement.

The advantage of this technique is that it is relatively resistant to image decay by opacities. Fig. 1 shows the difference in Logmar acuity between a group of cataract patients and normal controls. Fig. 2 shows that both groups have similar displacement thresholds, that is the sensitivity to movement detection is not impaired by moderate lens opacities. The test is quick and easy for the patient and there is very little inter-test variability. Preliminary results are encouraging and give a more accurate prediction of post-operative acuity than the laser interferometer, but the problem of false positives in the presence of foveal damage has not been resolved as the sensitivity for displacement threshold does not fall as steeply as Snellen acuity as the field of view becomes eccentric (Fig. 3).

As with laser interferometry a poor response is a good predictor of retinal damage; a good response should be judged in the light of other findings.

It can be seen from this brief survey that Snellen visual acuity alone is no longer an adequate measure of a cataract patient's disability. Tests of retinal function in the presence of cataract are helpful but need further refinement.

The data in Figs. 1 and 2 were supplied by Dr. Mark Hurst and Fig. 3 was constructed from experiments conducted by Dr. Terry Buckingham, both of the Clinical Vision Research Unit, Department of Optometry, Bradford University.

Key words: Cataract evaluation, Cataract surgery, Contrast sensitivity, Displacement thresholds, Retinal acuity, Visual acuity.

\section{REFERENCES}

1. Ferris FL, Kassof A, Bresnick GH, Bailey I. New visual acuity charts for clinical research. Am J Ophthalmol 1982; 94:91-6.

2. Elliott DB, Hurst MA, Weatherill JR. Comparing clinical tests of visual function in cataract with the patient's perceived visual disability. Eye 1990;4:712-17.

3. Pardhan S. Binocular performance in patients with unilateral cataract using the Regan test. Eye 1993;7:59-62

4. Faulkner W. Laser interoferometric prediction of postoperative acuity in patients with cataracts. Am J Ophthalmol 1983;95:626-36. 